\title{
Arquitectura de Raíces en Beilschmiedia miersii (Gay) Kosterm.
}

\author{
Peña-Rojas, Karen 1*; Donoso, Sergio 2; Espinoza, Claudia ${ }^{3}$; Gangas, Rodrigo 4; Quintanilla, Miguel 5; \\ Aravena, Marie Claire 6; Pozo, Rodrigo ${ }^{7}$; Sánchez, Juan ${ }^{8}$ y Martínez, Ema9 . \\ ${ }^{1}$ Doctora en Fisiología de Plantas y Ambiente, Académico e Investigadora en la Facultad de Ciencias Forestales y de la Conservación \\ de la Naturaleza (CFCN), Universidad de Chile, Santiago/Chile, kpenarojas@uchile.cl. \\ 2 Doctor Ingeniero de Montes. Académico e Investigador Académico en la Facultad de Ciencias Forestales y de la Conservación de \\ la Naturaleza (CFCN), Universidad de Chile, Santiago/Chile, sedonoso@uchile.cl. \\ ${ }^{3}$ Ingeniera Forestal. Investigadora en la Facultad de Ciencias Forestales y de la Conservación de la Naturaleza (CFCN), Universidad \\ de Chile, Santiago/Chile, cespinoza@ug.uchile.cl. \\ ${ }^{4}$ Ingeniero Forestal. Investigador en la Facultad de Ciencias Forestales y de la Conservación de la Naturaleza (CFCN), Universidad \\ de Chile, Santiago/Chile, rod.gangas@gmail.com. \\ ${ }^{5}$ Ingeniero Forestal. Investigador en la Facultad de Ciencias Forestales y de la Conservación de la Naturaleza (CFCN), Universidad \\ de Chile, Santiago/Chile, mig.quintanilla@gmail.com. \\ ${ }^{6}$ Ingeniera Forestal. Investigadora en la Facultad de Ciencias Forestales y de la Conservación de la Naturaleza (CFCN), Universidad \\ de Chile, Santiago/Chile, marie.aravena@ug.uchile.cl. \\ ${ }^{7}$ Licenciado en Ciencias Forestales, Consultor, Santiago/Chile, ro.pozo88@gmail.com. \\ ${ }^{8}$ Asesor de Biodiversidad, Gerencia de Sustentabilidad Operación El Soldado, Anglo American Sur S.A. Nogales/Chile, \\ juan.sanchez@angloamerican.com. \\ ${ }^{9}$ Gerenta de Sustentabilidad Operación El Soldado, Anglo American Sur S.A., ema.martinez@angloamerican.com. \\ *Autor de correspondencia
}

\section{RESUMEN}

Beilschmiedia miersii (belloto del norte) es un árbol siempreverde de la familia Lauraceae, endémico de Chile central. Para conocer su arquitectura de raíces, hasta ahora desconocida, se realizó la exploración del sistema radical en árboles adultos autorizados por el Servicio de Evaluación Ambiental (SEA) para ser trasplantados bajo la resolución de calificación ambiental. La evaluación se realizó en los sectores Infiernillo y Los Coiles, en la proximidad de la mina de cobre El Soldado (Anglo American, Chile), en la Región de Valparaíso. Se midió la altura, el DAP y el radio de copa en siete árboles. Posteriormente, se analizó el sistema radical, registrando la longitud, distancia geométrica, profundidad y orientación de las raíces de cada árbol. Las raíces alcanzaron profundidades medias entre 35 y $110 \mathrm{~cm}$, similar a otras especies arbóreas que exploran volúmenes superficiales del suelo. La distancia geométrica y la longitud de las raíces se correlacionó mejor con el DAP $\left(R^{2}=0,97\right)$ y con la altura $\left(R^{2}=0,94\right)$. El belloto del norte más grande medido $(68,6 \mathrm{~cm}$ de DAP y $21 \mathrm{~m}$ de altura), presentó la distancia máxima de raíz de $27 \mathrm{~m}$ y la longitud máxima de $36 \mathrm{~m}$. Se detectó que las raíces más profundas crecieron en dirección al curso de agua en la mayoría de los árboles. Las raíces más largas se orientaban en la dirección opuesta o paralelas al curso de agua. Los resultados son aplicables para definir zonas de protección de raíces y requerimientos de hábitat donde crece belloto del norte; también como referencia para otras especies sin antecedentes en esta materia.

Palabras clave: Beilschmiedia miersii, longitud de raíces, zona de protección de raíces, arquitectura de raíces.

\section{SUMMARY}

Beilschmiedia miersii (belloto del norte) is an evergreen tree from Lauraceae family, endemic to central Chile. There are no antecedents about the root architecture of this species. In order to know its root architecture, the root system was explored in mature trees authorized by the Environmental Evaluation Service (SEA) to be transplanted under an environmental qualification resolution (RCA). The evaluation was carried out in the Infiernillo and Los Coiles sectors, located near the EI Soldado copper mine (Anglo American, Chile) at the Valparaíso Region. The height, DBH and crown radius were measured in seven trees. Subsequently, root system was analyzed, measuring the length, geometric distance, depth and orientation of roots of each tree. Roots reached average depths between $35 \mathrm{~cm}$ and $110 \mathrm{~cm}$, similar to other tree species that explore surface volumes of soil. Geometric distance and root length were better correlated with $D B H\left(R^{2}=0.97\right)$ and height $\left(R^{2}=0.94\right)$. The largest tree of this specie measured $(68.6 \mathrm{~cm} \mathrm{DBH}$ and $21 \mathrm{~m}$ height), presented a maximum root distance of $27 \mathrm{~m}$ and a maximum length of $36 \mathrm{~m}$. The deepest roots were found to grow in the direction of the water stream in most of the trees. The longest roots were oriented in the opposite direction or parallel to the water stream. The results are applicable to define root protection zones and requirements habitat where "belloto del norte" grows. The study contributes as reference for species with similar characteristics that, likewise lack antecedents.

Key words: Beilschmiedia miersii, root length, root protection zone, root architecture. 


\section{INTRODUCCIÓN}

Beilschmiedia miersii (Gay) Kosterm (belloto del norte) es un árbol siempreverde, endémico de Chile Central. Generalmente se encuentra en fondos de quebradas, cerca de cursos de agua, laderas protegidas de exposición sur, sitios con influencia de neblina costera o pequeños valles de depositación creados en el ensanchamiento de pequeñas cuencas (Serra et al.,1986; Hechenleitner et al., 2005). Forma bosques en asociación con especies de ambientes húmedos, siendo la dominante en cuanto a tamaño y altura (Novoa, 2004; Cabello, 2013). Actualmente, está declarada Monumento Natural de Chile (DS No 13/1995) (Ministerio de Agricultura, 1995) y clasificada como Vulnerable a la extinción (Proceso 2 DS N 50/2008) ( Ministerio Secretaría General de la Presidencia, 2008).

En Chile se han publicado varios trabajos relacionados con esta especie. Se han hecho descripciones morfológicas de flores, frutos y hojas (Rodríguez et al., 1983, Serra et al., 1986), estudios de fenología (Donoso y Cabello, 1978), estudios de la estructura y dinámica de los bosques que conforma (Brito-Rozas y Flores-Toro, 2014) y sobre la regeneración natural (Morales et al., 2015; Kremer et al., 2019), entre otros. Incluso se han realizado trabajos de relocalización de ejemplares de $B$. miersii para evitar su eliminación frente a intervenciones industriales (Arellano y Morales, 2006). Sin embargo, hasta la fecha no existen antecedentes técnicos sobre la arquitectura de raíces en $B$. miersii. La escasa información que hay corresponde a observaciones de terreno que no están del todo documentadas.

El estudio de raíces en plantaciones y bosques naturales es un apoyo en la toma de decisiones respecto al manejo de la vegetación. Las raíces son el soporte de todo el crecimiento aéreo, y tienen un rol vital en el abastecimiento y almacenamiento de agua y nutrientes (Verdaguer y Molinas, 1992). Pueden funcionar como sensores de estrés hídrico, ante la cual envían señales bioquímicas a la parte aérea (Kramer y Boyer, 1995). Se ha indicado que las raíces de los árboles y arbustos son elementos estructurales importantes en la estabilidad del suelo, debido al refuerzo mecánico que adhieren y a la disminución de la presión de poros del suelo (Barrera et al., 2013). Y se ha demostrado la estrecha relación que existe entre el sistema de raíces de los árboles y algunas redes de hongos micorrízicos, que aumentan la capacidad de exploración en el suelo y puede facilitar la "comunicación" entre árboles (Simard, 2012; Simard et al., 2015; Pickles y Simard, 2017).

Las raíces varían en forma, tipo y función, presentando un alto grado de plasticidad morfológica incluso variando a nivel de especie. La arquitectura del desarrollo del sistema radical de un árbol se encuentra influenciada por factores genéticos y ambientales (Foxx et al., 1984; Kalliokosky et al., 2008). Los estudios de raíz se dividen en dos categorías, destructivos y no destructivos. El método destructivo (excavación) es la técnica más comúnmente utilizada, aunque no es el recomendado, ya que muchas veces el sitio dificulta las mediciones directas en las raíces, necesitándose una gran inversión de tiempo y recursos para realizar la tarea (Sanesi et al., 2013). Esta es una de las razones de los escasos estudios realizados en Chile sobre arquitectura de raíces, particularmente en especies del bosque nativo chileno (Giliberto y Estay, 1978). Los resultados de este trabajo permitirán complementar la información existente sobre B. miersii con énfasis en su sistema radical.

\section{OBJETIVOS}

Analizar la estructura del sistema radical en Beilschmiedia miersii, utilizando los árboles autorizados para ser trasplantados mediante la Resolución de Calificación Ambiental emitida el año 2004 de acuerdo a la Ley 19.300 sobre Bases Generales del Medio Ambiente de Chile (RCA N¹63/2004).

Los objetivos específicos son:

- Caracterizar dendrométricamente a los árboles de B. miersii.

- Analizar el sistema de raíces, determinando la distancia geométrica, longitud, profundidad y orientación de las raíces de cada árbol.

\section{MATERIAL Y MÉTODO}

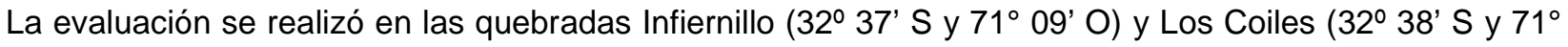
09' O), situadas en el sector de la mina EI Soldado, comuna de Nogales, Región de Valparaíso. Actualmente propiedad de Anglo American Sur S.A. 
Se expusieron las raíces de 7 individuos de B. miersii, (4 en Infiernillo y 3 en Los Coiles). A cada árbol se le midió la altura, el diámetro a la altura del pecho (DAP; a 1,3 m), y el radio de copa en orientación de los puntos cardinales (Norte-Sur-Este-Oeste).

Posteriormente, se realizó la prospección de raíces a través de la remoción manual del material edáfico alrededor de éstas. Se utilizaron chuzos, palas, espátulas, entre otras herramientas. Las rocas más grandes fueron movidas y trasladadas con una retroexcavadora.

En caso necesario, se ancló el individuo mediante cuerdas para evitar una eventual caída por viento (Figura 1).

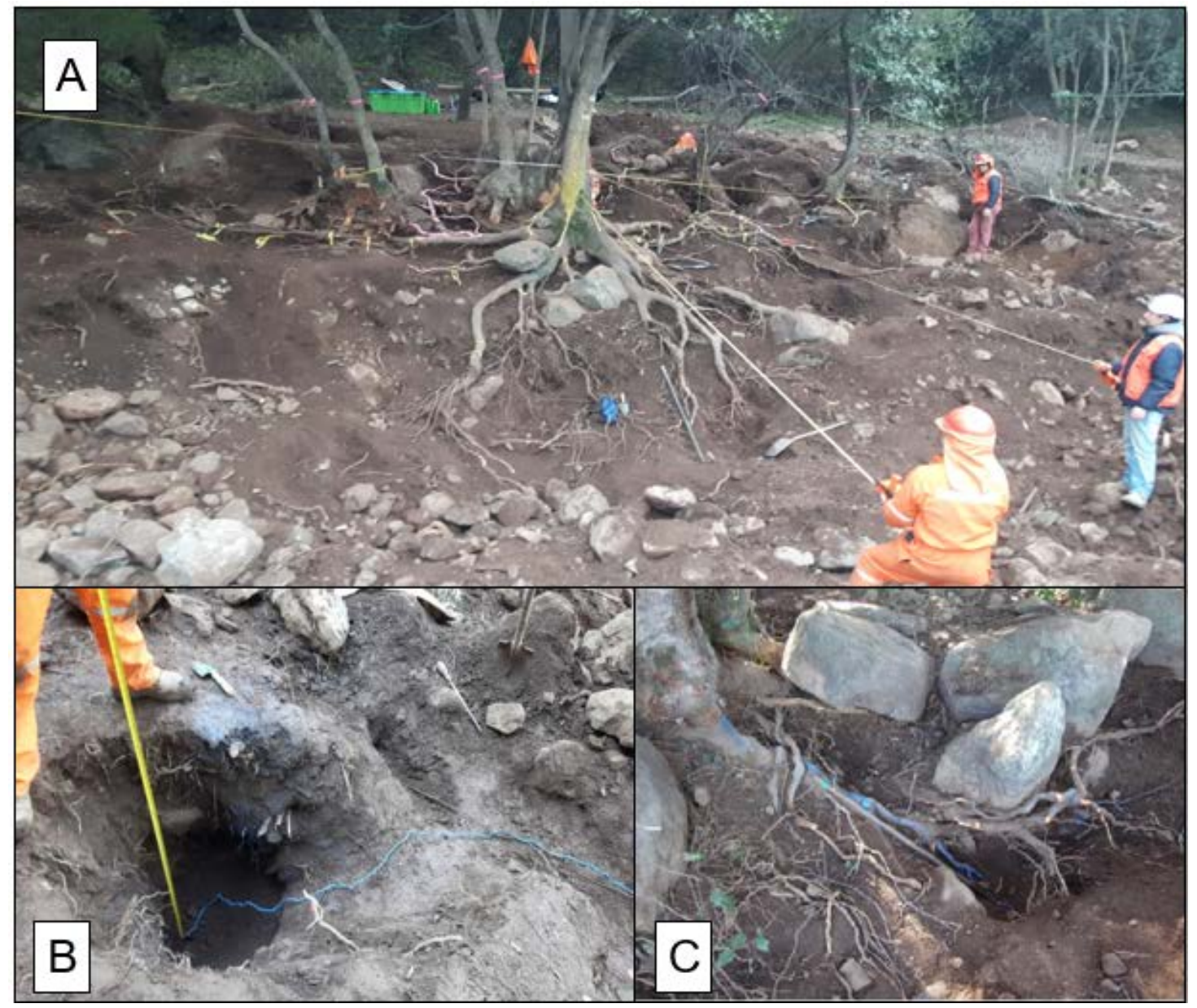

Figura 1. Medición in situ de raíces de Beilschmiedia miersii. Distancia de raíz (A), Profundidad de raíz (B) y Pedregosidad en el área de estudio (C).

Se hizo el seguimiento de cada una de las raíces principales desde el fuste hasta alcanzar un diámetro mínimo aproximado de $0,5 \mathrm{~cm}$. Para diferenciarlas, cada raíz fue marcada. Una vez efectuado el seguimiento fueron cubiertas con el material removido para evitar el desecamiento por sobreexposición. Se midió la longitud (largo que sigue el contorno de la raíz) y la distancia geométrica (distancia en línea recta que separa dos puntos del terreno) de las raíces.

Se registró la profundidad alcanzada por las raíces y, se determinó la orientación principal, entre el inicio de la raíz y el punto donde se encontraba el extremo más distante, mediante una brújula. Este último registro se proyectó en un plano para calcular el área de exploración de raíces $\left(\mathrm{m}^{2}\right)$. 


\section{RESULTADOS}

Los árboles evaluados, corresponden a individuos que previo al estudio, se encontraban vigorosos y sin problemas sanitarios. Las áreas de trabajo no habían sido sometidas a disturbios antrópicos. Las principales características de los árboles analizados se presentan en el Cuadro 1.

Cuadro 1. Características dendrométricas de los árboles estudiados y proporción del sistema de raíces analizado.

\begin{tabular}{|c|c|c|c|c|c|c|c|c|}
\hline \multirow{2}{*}{ Sector } & \multirow{2}{*}{ Árbol } & \multirow{2}{*}{$\begin{array}{c}\text { Altura } \\
(\mathrm{m})\end{array}$} & \multirow{2}{*}{$\begin{array}{l}\text { DAP } \\
(\mathrm{cm})\end{array}$} & \multicolumn{4}{|c|}{$\begin{array}{c}\text { Radio de Copa } \\
\text { (m) }\end{array}$} & \multirow{2}{*}{$\begin{array}{c}\text { Proporción } \\
\text { Analizada } \\
(\%)^{*}\end{array}$} \\
\hline & & & & Norte & Sur & Este & Oeste & \\
\hline \multirow{4}{*}{ Infiernillo } & 1 & 8,5 & 17,5 & 4,75 & 3,05 & 3,15 & 5,30 & 100 \\
\hline & 2 & 8,5 & 15,6 & 5,70 & 5,10 & 3,35 & 3,20 & 100 \\
\hline & 3 & 7,0 & 17,5 & 0,50 & 4,20 & 0,55 & 5,45 & 100 \\
\hline & 4 & 11,5 & 24,4 & 3,45 & 6,55 & 5,40 & 3,40 & 100 \\
\hline \multirow{3}{*}{ Los Coiles } & 5 & 3,2 & 3,1 & 0,68 & 0,88 & 0,62 & 1,08 & 100 \\
\hline & 6 & 19,6 & 48,5 & 6,87 & 4,21 & 4,49 & 4,63 & 100 \\
\hline & 7 & 21,0 & 68,6 & 9,47 & 8,05 & 6,16 & 6,61 & 80 \\
\hline
\end{tabular}

Las raíces de $B$. miersii eran levemente estriadas, de color rojizo y sin olor. En el lugar se observaron rocas de gran volumen que influyeron en la arquitectura de raíces. Al encontrarse con estas rocas, las raíces redujeron su diámetro para avanzar tanto en profundidad como en longitud. Los resultados obtenidos para cada árbol se presentan en el Cuadro 2, donde se indican los valores medios para cada variable.

Cuadro 2. Valores medios \pm error estándar, de longitud, diámetro y valor máximo de profundidad de raíz según árbol.

\begin{tabular}{lccccccc}
\hline \multirow{2}{*}{ Parámetro } & \multicolumn{7}{c}{ Árboles } \\
\cline { 2 - 8 } & $\mathbf{1}$ & $\mathbf{2}$ & $\mathbf{3}$ & $\mathbf{4}$ & $\mathbf{5}$ & $\mathbf{6}$ & $\mathbf{7}$ \\
\hline Longitud media $(\mathrm{m})$ & $7,58 \pm 0,22$ & $5,14 \pm 0,30$ & $3,72 \pm 0,80$ & $6,36 \pm 0,22$ & $1,86 \pm 0,17$ & $14,52 \pm 0,37$ & $20,98 \pm 0,78 *$ \\
\hline Distancia media $(\mathrm{m})$ & $5,51 \pm 0,19$ & $3,93 \pm 0,22$ & $2,61 \pm 0,53$ & $4,81 \pm 0,17$ & $1,36 \pm 0,11$ & $10,95 \pm 0,30$ & $15,03 \pm 0,64$ * \\
\hline Diámetro inicial $(\mathrm{cm})$ & $12,45 \pm 2,31$ & $14,33 \pm 1,51$ & $9,05 \pm 2,09$ & $20,13 \pm 4,7$ & $1,22 \pm 0,24$ & $20,13 \pm 4,70$ & $30,66 \pm 5,01 *$ \\
\hline Diámetro final $(\mathrm{cm})$ & $0,40 \pm 0,04$ & $0,38 \pm 0,02$ & $0,42 \pm 0,04$ & $0,37 \pm 0,01$ & $0,16 \pm 0,02$ & $0,5 \pm 0,03$ & $0,74 \pm 0,07$ \\
\hline Profundidad media $(\mathrm{m})$ & $0,80 \pm 0,04$ & $0,94 \pm 0,04$ & $1,1 \pm 0,11$ & $0,62 \pm 0,02$ & $0,35 \pm 0,07$ & $0,52 \pm 0,02$ & $0,62 \pm 0,03$ * \\
\hline Profundidad máxima $(\mathrm{m})$ & 2,05 & 1,50 & 1,50 & 1,55 & 1,48 & 1,62 & 1,53 * \\
\hline * Resultados con
\end{tabular}

* Resultados con el análisis del 80\% de las raíces del árbol.

Se observó una relación positiva entre la distancia y longitud promedio de las raíces, con la altura, el diámetro a la altura del pecho (DAP) y el diámetro de copa de los árboles estudiados, pero esta correlación fue más alta con las dos primeras variables (Figura 2).

Se analizó la longitud y distancia, media y máxima, de las cinco raíces de mayor extensión en los cinco árboles de mayor tamaño (Cuadro 3). Para el caso del árbol siete, solo se analizó el $80 \%$ de sus raíces, debido a la presencia de una gran roca que dificultaba la continuidad de la tarea. Con la información de los diámetros iniciales de estas raíces, se pudo estimar una longitud máxima de 36,0 m. 
Ciencia \& Investigación Forestal Vol. 27 N². Agosto 2021

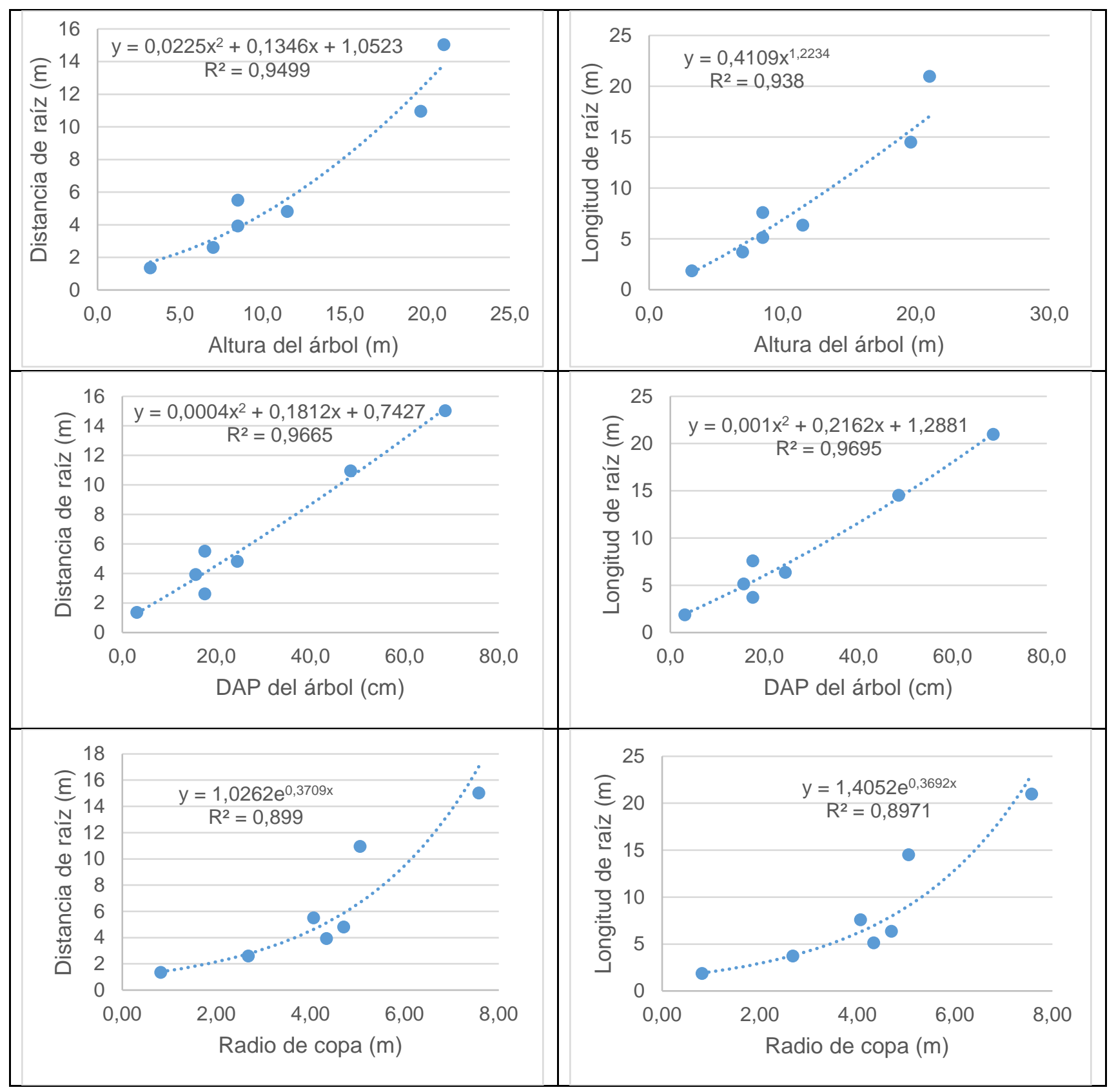

Figura 2. Correlación entre distancia y longitud de las raíces con la altura total, DAP y radio de copa de belloto del Norte.

Cuadro 3. Longitud y distancia media y máxima, de las cinco raíces de mayor tamaño por árbol.

\begin{tabular}{cccccc}
\hline Árbol & $\begin{array}{c}\text { DAP } \\
\mathbf{( c m})\end{array}$ & $\begin{array}{c}\text { Longitud } \\
\text { media } \\
(\mathbf{m})\end{array}$ & $\begin{array}{c}\text { Longitud } \\
\text { máxima } \\
(\mathbf{m})\end{array}$ & $\begin{array}{c}\text { Distancia } \\
\text { media } \\
(\mathbf{m})\end{array}$ & $\begin{array}{c}\text { Distancia } \\
\text { máxima } \\
(\mathbf{m})\end{array}$ \\
\hline $\mathbf{1}$ & 17,5 & $15,6 \pm 1,3$ & 17,6 & $13,4 \pm 0,6$ & 15,4 \\
\hline $\mathbf{2}$ & 15,6 & $16,7 \pm 3,5$ & 20,3 & $15,9 \pm 1,6$ & 20,2 \\
\hline $\mathbf{4}$ & 24,4 & $15,1 \pm 0,3$ & 15,9 & $14,2 \pm 0,9$ & 16,5 \\
\hline $\mathbf{6}$ & 48,5 & $26,5 \pm 0,6$ & 28,3 & $20,8 \pm 0,2$ & 21,2 \\
\hline $\mathbf{7}$ & 68,6 & $35,0 \pm 0,3^{\star}$ & $36,0^{*}$ & $26,3 \pm 0,2$ & 27,0 \\
\hline
\end{tabular}

Los valores corresponden a medias \pm desviación estándar. * Resultados con el análisis del $80 \%$ de las raíces del árbol. Área de Exploración y Orientación de Raíces 
El área de exploración de raíces fue mayor en los individuos 6 y 7, con 527 y $498 \mathrm{~m}^{2}$, respectivamente. Particularmente, los árboles 1 y 3 que tienen el mismo DAP $(17,5 \mathrm{~cm})$, presentaron áreas de exploración muy diferentes (124,5 y 6,9 $\mathrm{m}^{2}$, respectivamente). Esto puede explicarse por la abundante pedregosidad superficial existente en el lugar donde crecía el individuo 3. Al trazar una tendencia lineal entre los datos, se encontró una correlación positiva entre el área de exploración de raíces y la altura $\left(R^{2}=0,91\right)$, y entre el área de exploración de raíces y el DAP $\left(R^{2}=0,87\right)$. Los árboles 4,2 , y 5 presentaron un área de exploración de $98,3,51,8$ y $12,0 \mathrm{~m}^{2}$, respectivamente.

Al analizar las raíces de $B$. miersii en función de la orientación, el parámetro más afectado correspondió a la profundidad de las raíces, con un desarrollo mayor hacia el cuadrante 2 ( 1 a 90º, dirección Este, donde se encuentra el curso de agua) en cuatro de los siete individuos analizados. Menos claro fue el parámetro de longitud, donde en algunos casos las raíces más largas se orientaban en la dirección opuesta al curso de agua y en otras ocasiones de forma paralela a este (Cuadro 4; Figura 3). Por lo tanto, la orientación de longitud de las raíces de $B$. miersii, no necesariamente, se condice con las áreas donde se alcanza la mayor profundidad.

Cuadro 4. Orientación de las raíces de B. miersii.

\begin{tabular}{|c|c|c|c|c|}
\hline Sector & Árbol & Cuadrante & $\begin{array}{c}\text { Longitud } \\
\text { media } \\
(\mathrm{m})\end{array}$ & $\begin{array}{l}\text { Profundidad } \\
\text { media } \\
\text { (m) }\end{array}$ \\
\hline \multirow{16}{*}{ Infiernillo } & \multirow{4}{*}{1} & $1(N-E)$ & $6,72 \pm 0,32$ & $1,05 \pm 0,06$ \\
\hline & & $2(S-E)$ & $7,40 \pm 0,29$ & $0,99 \pm 0,07$ \\
\hline & & $3(\mathrm{~s}-\mathrm{O})$ & $7,21 \pm 0,43$ & $0,90 \pm 0,07$ \\
\hline & & $4(\mathrm{~N}-\mathrm{O})$ & $8,70 \pm 0,27$ & $0,76 \pm 0,07$ \\
\hline & \multirow{4}{*}{2} & $1(N-E)$ & $5,41 \pm 0,29$ & $1,18 \pm 0,10$ \\
\hline & & 2 (S-E) & $4,66 \pm 0,32$ & $0,94 \pm 0,03$ \\
\hline & & $3(\mathrm{~S}-\mathrm{O})$ & $5,55 \pm 0,50$ & $0,76 \pm 0,07$ \\
\hline & & $4(\mathrm{~N}-\mathrm{O})$ & $5,53 \pm 0,26$ & $0,87 \pm 0,11$ \\
\hline & \multirow{4}{*}{3} & $1(N-E)$ & - & - \\
\hline & & 2 (S-E) & $3,30 \pm 0,56$ & $1,14 \pm 0,09$ \\
\hline & & $3(\mathrm{~S}-\mathrm{O})$ & $4,60 \pm 0,10$ & $1,50 \pm 0,0$ \\
\hline & & $4(\mathrm{~N}-\mathrm{O})$ & $0,50 \pm 0,00$ & $0,4 \pm 0,00$ \\
\hline & \multirow{4}{*}{4} & $1(N-E)$ & $5,47 \pm 0,26$ & $0,82 \pm 0,06$ \\
\hline & & 2 (S-E) & $5,52 \pm 0,18$ & $0,85 \pm 0,05$ \\
\hline & & $3(\mathrm{~S}-\mathrm{O})$ & $5,26 \pm 0,22$ & $0,71 \pm 0,04$ \\
\hline & & $4(\mathrm{~N}-\mathrm{O})$ & $8,72 \pm 0,53$ & $0,57 \pm 0,05$ \\
\hline \multirow{12}{*}{ Los Coiles } & \multirow{4}{*}{5} & $1(N-E)$ & $1,69 \pm 0,29$ & $0,23 \pm 0,05$ \\
\hline & & $2(S-E)$ & $2,33 \pm 0,30$ & $0,70 \pm 0,19$ \\
\hline & & $3(\mathrm{~S}-\mathrm{O})$ & $1,91 \pm 0,33$ & $0,27 \pm 0,03$ \\
\hline & & $4(\mathrm{~N}-\mathrm{O})$ & $1,21 \pm 0,38$ & $0,12 \pm 0,02$ \\
\hline & \multirow{4}{*}{6} & $1(N-E)$ & $6,57 \pm 0,54$ & $0,32 \pm 0,02$ \\
\hline & & $2(S-E)$ & $17,48 \pm 0,30$ & $0,60 \pm 0,03$ \\
\hline & & $3(\mathrm{~S}-\mathrm{O})$ & $12,12 \pm 0,91$ & $0,46 \pm 0,04$ \\
\hline & & $4(\mathrm{~N}-\mathrm{O})$ & $5,49 \pm 0,45$ & $0,28 \pm 0,02$ \\
\hline & \multirow{4}{*}{7} & $1(N-E)$ & $9,63 \pm 1,49$ & $0,87 \pm 0,27$ \\
\hline & & 2 (S-E) & $13,62 \pm 0,49$ & $0,58 \pm 0,05$ \\
\hline & & $3(\mathrm{~S}-\mathrm{O})$ & $26,00 \pm 1,27$ & $0,58 \pm 0,03$ \\
\hline & & $4(\mathrm{~N}-\mathrm{O})$ & $22,44 \pm 0,95$ & $0,72 \pm 0,08$ \\
\hline
\end{tabular}

Los valores corresponden a medias \pm error estándar. 


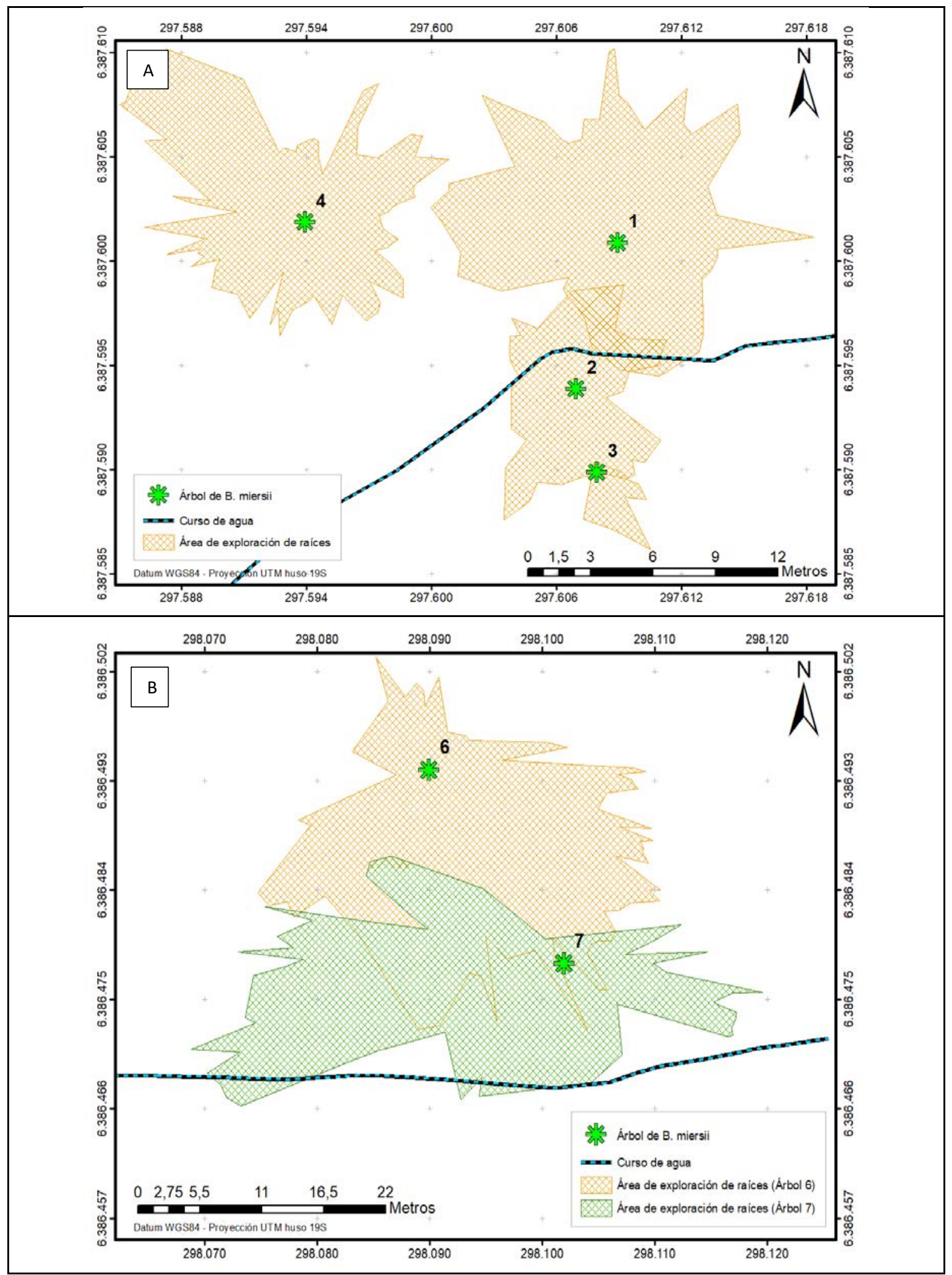

Figura 3. Representación del área de exploración de raíces y su orientación, en Infiernillo (A) y Los Coiles (B). 


\section{DISCUSIÓN}

La arquitectura de raíz generalmente se refiere a la configuración espacial del sistema subterráneo, es decir, al tamaño y la ubicación explícita de las partes leñosas radicales (Kalliokoski et al., 2008). Aunque la idea más difundida es que el desarrollo de una raíz se extienda en profundidad, lo cierto es que existe una importante tendencia a que las raíces exploren volúmenes superficiales del suelo (Dobson, 1995; Rizzardi y Calvo, 2019). Algunas referencias señalan que la mayoría de las raíces se encuentran en los primeros $50 \mathrm{~cm}$ del perfil, y gran parte de la actividad de las raíces y micorrizas se realiza en los primeros $20 \mathrm{~cm}$, dependiendo de la aireación y fertilidad del suelo (Donoso et al., 2002). Otros autores indican que entre el 80 y $90 \%$ del total de las raíces de los árboles se concentrarían en los primeros $100 \mathrm{~cm}$ del perfil del suelo (Perry, 1989; Crow, 2005; Rizzardi y Calvo, 2019). Debido a que el oxígeno suele encontrarse en la capa superior del suelo, la mayor concentración de raíces nutríferas o conductoras se presenta en esta zona. Otra característica importante a considerar es que las raíces, según su morfología y tamaño, tienen diferentes funciones (Eissenstat et al., 2000) las que van cambiando a lo largo de la vida del árbol. Cuando las plantas perciben estrés por déficit de agua, las raíces tienden a seguir creciendo y penetrar en capas más profundas del suelo (Hoogenboom et al., 1987; Sponchiado et al., 1989). Las raíces profundas se componen en gran parte de raíces finas (Eissenstat et al., 2000). Aún existe una falta de consenso sobre qué se considera como "raíz fina". Aunque muy a menudo se considera que las raíces finas son raíces $<2$ $\mathrm{mm}$ de diámetro, las raíces $<0,2 \mathrm{~mm}$ de diámetro pueden representar más del $50 \%$ de la longitud total de la raíz (Pallant et al., 1993; Amato y Pardo, 1994; Pierret et al.,2005). En este estudio, la excavación permitió un análisis de raíces, exponiéndolas hasta un diámetro de $0,5 \mathrm{~cm}$ lo que reduce la proporción de raíz fina, en los individuos de $B$. miersii, a los extremos finales de las raíces. Por lo tanto, se comparte el planteamiento que zonas subterráneas de la planta se expanden, principalmente de forma lateral, formando un plato que aumenta en circunferencia con el desarrollo del árbol (Moore, 2003). Pese a ello, Schenk y Jackson (2002) indican que, incluso cuando se consideren los factores externos en la modelación de la arquitectura de las raíces, las profundidades de muestreo a menudo se deciden de manera arbitraria, abordando valores que son demasiado superficiales para permitir estimaciones confiables de la profundidad de enraizamiento.

Se ha documentado en especies adaptadas a ambientes secos, que ellas presentan sistemas radiculares duales, con raíces laterales poco profundas que explotan pequeños eventos de lluvia que apenas penetran el suelo, y además con raíces profundas que aprovechan fuentes de agua profunda (Canadell y Zedler 1995). Otros autores indican que frente a la escasez hídrica del suelo, las plantas aumentan la densidad radicular penetrando a mayor profundidad (Hoogenboom et al., 1987; Sponchiado et al., 1989). Por lo tanto, la profundidad de enraizamiento tiene directa relación con la capacidad de las plantas para extraer agua del suelo en profundidad (Padilla y Pugnaire, 2007). Esta característica sería esencial para especies esclerófilas de clima mediterráneo, que deben mantener el estado hídrico fundamental durante toda la temporada estival (Rundel et al., 2019) y ahora incluso, durante los secos inviernos.

B. miersii es una especie altamente dependiente del agua edáfica (Amigo y Flores-Toro, 2012), de raíces poco profundas o superficiales (shallow-rooted) como Cryptocarya alba (Ovalle et al., 2015), con la característica principal de ser menos tolerantes a eventos de sequía. Las raíces superficiales se caracterizan por concentrar la producción de raíces finas en los primeros $40 \mathrm{~cm}$ del suelo (Hoffmann y Kummerow, 1978; Machado et al., 2017). Cameron (1963) describe en su estudio de Beilschmiedia tawa (A. Cunn.) Benth. et Hook. F. ex Kirk como una masa de raíces de tamaño relativamente uniforme integradas en la parte superior y cercana al fuste (shallow-rooted), y con raíces laterales (deep-rooted) que se extienden hasta descender oblicua y verticalmente. Se dificulta la identificación de una raíz principal.

El desarrollo de las raíces en profundidad, B. miersii comparte características con otras especies nativas y exóticas. Por ejemplo, Hoffmann y Kummerow (1978) encontraron individuos de Lithraea caustica (Molina) Hook. et Arn. que desarrollaban raíces gruesas a más de $60 \mathrm{~cm}$ de profundidad. Comparativamente, Cryptocarya alba (Molina) Looser tenía un enraizamiento menos profundo y Colliguaja odorifera Molina tenía un sistema de raíces poco profundo con raíces finas en la capa de suelo de 20 a $40 \mathrm{~cm}$. Watson y O'Loughlin (1990), en un estudio realizado en Pinus radiata D. Don, encontraron que todas las raíces laterales se limitaron al metro superior del perfil del suelo, y hasta un $75 \%$ ocurrió en la zona de 0-50 cm. Los árboles de $B$. miersii evaluados, alcanzaron profundidades medias de entre 35 y $110 \mathrm{~cm}$. Extraño es el comportamiento distante que posee con respecto a un individuo maduro de $B$. tawa, estudiado en Nueva Zelanda, donde la profundidad del sistema radical fue de 1 a $2 \mathrm{~m}$, siendo menos profundos en suelos más 
compactos (Cameron, 1963). Este comportamiento podría estar influenciado por factores externos al crecimiento de la raíz como la profundización de la humedad a lo largo del perfil del suelo (Padilla y Pugnaire, 2007). Lo cierto es que los sistemas de raíces poco profundos, presentan un alto estrés hídrico y el cese del crecimiento durante los meses de verano (Rundel et al, 2019; Giliberto y Estay 1978, Poole y Miller 1978, Hoffman y Kummerow, 1978).

Se observó que la distancia y la longitud promedio de las raíces se correlaciona mejor con el DAP y con la altura. Esto coincide con lo observado en ciertas excavaciones, donde la extensión de las raíces se correlacionó con la altura del árbol (Gilman, 1988; Rizzardi y Calvo, 2019). Igualmente, Kraemer et al. (1996) indicaron que la altura del árbol resulta en un buen predictor de la biomasa, la longitud, la penetración de la raíz principal y la extensión de la raíz lateral, en Juniperus occidentalis Hook. $\left(R^{2}\right.$ de 0,82 a 0,96). Sin embargo, Pierret et al. (2016) señalan que no existe una base sólida para asumir un rango estrecho de proporcionalidad entre la altura del dosel y la profundidad máxima de enraizamiento entre las especies. Por otra parte, Thomas (2014) indica que, en los árboles de bosques templados, la extensión total de raíces suele ser de 2 a 3 veces el radio de la copa, e incluso hasta 4 veces en suelos arenosos secos. Según este autor, y como alternativa, la extensión de las raíces se puede estimar como 1,5 a 2 veces la altura del árbol. Muy diferente a lo observado por Cameron (1963) con la excavación de un individuo maduro de $B$. tawa, que exhibió un sistema radical denso, compacto desarrollado lateralmente con 3 a $5 \mathrm{~m}$ de radio, lo que representa menos de la mitad del radio promedio de copa. En el caso de $B$. miersii, y considerando la distancia máxima de las raíces más largas registradas en este estudio, la extensión podría acercarse a 3,5 veces el radio promedio de copa, y 1,5 veces la altura del árbol. En la práctica, el radio promedio de copa podría usarse como una variable independiente, pero implica mayor cantidad de datos a registrar y su correlación es menor a la encontrada con el DAP y la altura del árbol en este estudio. La distribución de las raíces es un factor relacionado con las especies individuales, pero, en términos de proyección horizontal, el área de la raíz siempre excede el área de la copa (Sanesi et al., 2013). La estimación del radio de copa no es un predictor certero a menos que se establezca una relación para una especie en particular. Incluso entonces, estas relaciones pueden no ser válidas para árboles maduros (Day et al., 2010)

La distancia máxima de raíz encontrada para un belloto del norte de $68,6 \mathrm{~cm}$ de DAP y $21 \mathrm{~m}$ de altura fue de $27 \mathrm{~m}$ y una longitud máxima de $36 \mathrm{~m}$. Comparativamente, esta extensión de raíz es mayor a otros casos encontrados. Por ejemplo, en árboles de $P$. radiata de DAP 20, 45 y $60 \mathrm{~cm}$, se han encontrado extensiones de 3, 6 y $10 \mathrm{~m}$ aproximadamente en cada caso. Las raíces laterales tenían una longitud máxima de 4,7; 6,4 y $10,4 \mathrm{~m}$ a las edades de 8, 16 y 25 años, respectivamente (Watson y O'Loughlin, 1990). En J. occidentalis se han encontrado raíces laterales a 5,7 m del tronco, ocupando $102 \mathrm{~m}^{2}$ de suelo y concentradas en los $25 \mathrm{~cm}$ superiores del perfil. Otro estudio, en un ejemplar de Quercus suber L, de 40 $\mathrm{cm}$ de diámetro, $13 \mathrm{~m}$ de altura y 90 años de edad, describe un sistema radical periférico de unos $6 \mathrm{~m}$ aproximadamente, donde las raíces gruesas del árbol se distribuían laxamente y predominaban las raíces finas (Verdaguer y Molinas, 1992). Algunos ejemplares de roble (Quercus), álamo (Populus) o arce rojo (Acer rubrum $\mathrm{L}$ ) pueden presentar raíces laterales de hasta $30 \mathrm{~m}$ de desde el tronco. Otras especies, como Fraxinus excelsior $\mathrm{L}$. tienen raíces pioneras largas, de rápido crecimiento y moderadamente ramificadas, que pueden alcanzar hasta cuatro veces el radio de copa (Thomas, 2014), similar a lo encontrado en $B$. miersii.

La longitud y distancia de las raíces encontradas en este estudio sirven de referencia para definir la Zona de Protección de Árboles (Tree Protection Zone; TPZ) o Zona de Protección de Raíces (Root Protection Zone; RPZ) (Milandinovic, 2016), basado en el diámetro del árbol. Este espacio corresponde al área crítica o mínima de enraizamiento del árbol, donde al realizar cualquier construcción o intervención se podrían dañar las raíces y afecta la salud de los árboles. Resulta interesante plantear la necesidad de incluir este aspecto en la planificación de actividades de desarrollo que impliquen pérdida o daño a los árboles. La habilidad de una planta de modificar las características de sus raíces puede ser un mecanismo clave para evitar el estrés déficit o estrés hídrico (Olmo et al., 2014).

El crecimiento direccional de las raíces en relación a la disponibilidad de agua se conoce como hidrotropismo y comienza en la cofia con la percepción del gradiente de humedad (Cassab y Sanchez, 2006). Por mucho tiempo se ha aceptado que en la mayoría de los suelos las raíces son bastante superficiales, ya que las condiciones del subsuelo son a menudo poco favorables a su desarrollo, anaerobio y con barreras físicas como por ejemplo la roca madre (Moore, 2003). Otros autores, señalan que las raíces 
laterales en la mayoría de las plantas comienzan creciendo horizontalmente y luego, eventualmente, giran para crecer en o cerca de la vertical (Malamy, 2005). Pese a las discrepancias, la mayoría de los modelos de crecimiento de raíces de árboles asumen una densidad de raíces finas decreciente con la profundidad y la distancia desde el tallo del árbol (Mulia y Dupraz, 2006).

Al analizar las raíces de $B$. miersii en función de la orientación, la variable más afectada corresponde a la profundidad, con un desarrollo radical mayor hacia el Este en la mayoría de los árboles, justamente en dirección hacia el curso de agua. Menos claro fue el parámetro de longitud, donde en algunos casos las raíces más largas se orientaban en la dirección opuesta al curso de agua, y en otras ocasiones de forma paralela y en favor de la pendiente. Teóricamente, esta respuesta podría estar asociada a la búsqueda de agua de manera más gradual. Si bien $B$. miersii es una especie que crece en ambientes húmedos, no sería una especie adaptada a suelos saturados (hidrófita), que crezca sumergida parcial o totalmente en agua. Por ello, el movimiento de la mayoría de sus raíces será hacia zonas favorables, evitando las condiciones de anoxia o hipoxia que podrían existir en las áreas de inundación o donde la presión física que ejerce el agua pudiera dañar las raíces (Moreno et al., 2020). Los resultados y lo observado en terreno, muestran que el sistema de raíces privilegia estar en áreas húmedas, presentado una proporción pequeña de raíces que se encuentran en el área por donde escurre el agua. Se necesitan más estudios relacionados para desarrollar esta idea.

\section{CONCLUSIONES}

Los resultados observados en $B$. miersii concuerdan con lo descrito en otras especies arbóreas, donde las raíces exploran volúmenes superficiales del suelo, principalmente en los primeros $100 \mathrm{~cm}$ de profundidad. EI DAP y la altura del árbol son buenos indicadores para estimar la distancia y la longitud de las raíces en B. miersii.

Para un belloto del norte de $68,6 \mathrm{~cm}$ de DAP y $21 \mathrm{~m}$ de altura, la distancia máxima de raíz fue de $27 \mathrm{~m}$ y la longitud máxima de $36 \mathrm{~m}$. Los resultados son aplicables para definir zonas de protección en lugares donde crece belloto del norte. También pueden servir de referencia para especies de características similares.

Las raíces más profundas crecieron en dirección al curso de agua en la mayoría de los árboles. Menos claro fue el parámetro de longitud, donde en algunos casos las raíces más largas se orientaban en la dirección opuesta al curso de agua (pendiente arriba) y en otras ocasiones de forma paralela a este. Se recomienda seguir desarrollando estudios de este tipo y comparar estos resultados con los de otras especies nativas de Chile central.

\section{REFERENCIAS}

Amato, M. \& Pardo, A. (1994). Root length and biomass losses during sample preparation with different screen mesh sizes. Plant and Soil, 161(2): 299-303. https://doi.org/10.1007/BF00046401.

Amigo, J. \& Flores-Toro, L. (2012). Revisión sintaxonómica de los bosques esclerófilos de Chile Central: la alianza Cryptocaryon albae. Lazaroa., 33. Pp: 171-196. ISSN: 0210-9778.

Arellano, E. \& Morales, N. (2006). Traslado de árboles adultos: Al rescate del Belloto del Norte. Revista Agronomía y Forestal UC, 29. Pp: 26-29.

Barrera, J., Rivera, J. \& Cadena, M. (2013). Influencia del sistema radical de cuatro especies vegetales en la estabilidad de laderas a movimientos masales. Revista Cenicafé, 64(2): 59-77. ISSN: 01200275.

Brito-Rozas, E. \& Flores-Toro, L. (2014). Estructura y dinámica de los bosques de belloto el norte (Beilschmiedia miersii) de la Cordillera El Melón, comuna de Nogales, región de Valparaíso, Chile. Bosque, 35(1): 13-21. https://doi.org/10.4067/S0717-92002014000100002.

Cabello, Á. (2013). Beilschmiedia miersii (Gay) Kosterm, Belloto del Norte, Familia: Lauraceae. En: Donoso, C. (Ed). Las especies arbóreas de los bosques templados de Chile y Argentina. Autoecología. Marisa Cuneo Ediciones. Segunda Edición. Valdivia, Chile. Pp: 181-186. ISBN: 9789567173310.

Cameron, R.J. (1963). A study of the rooting habits of rimu and tawa in pumice soils. New Zealand journal of forestry, 8(5): 771-785.

Canadell, J. \& Zedler, P.H. (1995). Underground Structures of Woody Plants in Mediterranean Ecosystems of Australia, California, and Chile. En: Arroyo, M.T.K., Zedler, P.H. \& Fox, M.D. (Eds). Ecology and Biogeography of 
Mediterranean Ecosystems in Chile, California, and Australia. Ecological Studies (Analysis and Synthesis), 8. Pp: 177-210. ISBN: 9781461224907.

Cassab, G. \& Sánchez, Y. (2006). Diferenciación y Crecimiento Diferencial: La Capacidad Motriz de Las Plantas. En: Squeo, F.A. \& Cardemil, L. (Eds). Fisiología Vegetal. Ediciones Universidad de La Serena. La Serena, Chile. Capítulo XVII (17): 1-26.

Crow, P. (2005). The Influence of Soils and Species on Tree Root Depth. Environmental and Human Sciences Division. Forest Research Alice Holt Lodge. Farnham Surrey. Forestry Commission. Edinburgh. UK. ISSN: 14603802.

Day, S. D., Wiseman, E., Dickinson, S., \& Harris, J. Roger. (2010). Contemporary concepts of root system architecture of urban trees. Arboriculture \& Urban Forestry, 36(4): 149-159.

Dobson, M. (1995). Tree root system. Arboriculture Research and Information note. AAIS - Arboricultural Advisory and Information Service. Note 130/95/ARB.

Donoso, C. \& Cabello, Á. (1978). Antecedentes fenológicos y de germinación de especies leñosas chilenas. Ciencias Forestales, 1(2): 31-41.

Donoso, S., Ruíz, F. \& Herrera, M. (2002). Distribución y cantidad de biomasa de raíces finas en plantaciones clonales de Eucalyptus globlus. Ciencias Forestales, 16(1-2): 3-10.

Eissenstat D.M., Wells C.E., Yanai R.D. \& Whitbeck J.L. (2000). Building roots in a changing environment: implications for root longevity. New Phytol., 147. Pp: 33-42. https://doi.org/10.1046/j.1469-8137.2000.00686.x.

Foxx, T., Tierney, G. \& Williams, J. (1984). Rooting Depths of Plants Relative to Biological and Environmental Factors. Los Alamos, New Mexico 87545. U.S. Government Printing Office.

Giliberto, J. \&y Estay, H. (1978). Seasonal water stress in some Chilean matorral shrubs. Botanical Gazette. 139(2): 236-240. https://doi.org/10.1086/336995.

Gilman, E. (1988). Predicting root spread from trunk diameter and branch spread. Arboricultural Journal, 13:1, 25-32. Journal of Arboriculture. 14(4). 85-89. https://doi.org/10.1080/03071375.1989.9756398.

Hechenleitner, P., Gardner, M., Thomas, P., Echeverría, C., Escobar, B., Brownless, P. \& Martínez, C. (2005). Plantas Amenazadas del Centro-Sur de Chile. Distribución, Conservación y Propagación. Primera Edición. Universidad Austral de Chile y Real Jardín Botánico de Edimburgo. Valdivia, Chile. 188 p.

Hoffmann, A. \& Kummerow, J. (1978). Root studies in the Chilean matorral. Oecologia, 32(1): 57-69. https://doi.org/10.1007/BF00344689.

Hoogenboom, G., Peterson, C., \& Huck, M.G. (1987). Shoot growth rate of soybean as affected by drought stress.Agronomy Journal, 79(4): 598-607. https://doi.org/10.2134/agronj1987.00021962007900040003x.

Kalliokoski, T., Nygren, P. \& Sievänen, R. (2008). Coarse root architecture of three boreal tree species growing in mixed stands. Silva Fenica, 42(2): 189-210. https://doi.org/10.14214/sf.252.

Kramer, P. \& Boyer, J. (1995). Water relations of plants and soils. Chapter 5. Roots and Root Systems. Department of Botany. Duke University, Durhan, North Carolina. Academic Press. San Diego, California, USA. Pp: 115-166.

Kraemer, S., Miller P.M. \& Eddleman L.E. (1996). Root system morphology and development of seedling and juvenile Juniperus occidentalis. Forest Ecology and Management, 86(1-3): 229-240. https://doi.org/10.1016/S03781127(96)03769-3.

Kremer, K., Promis, Á., Mancilla, G. \& Magni, C. (2019). Leaf litter and irrigation can increase seed germination and early seedling survival of the recalcitrant-seeded tree Beilschmiedia miersii. Austral Ecology, 44. Pp: 86-94. https://doi.org/10.1111/aec.12655.

Machado, B., Magro, M., Silva de Souza, D., Rufato, L. \& Anneliese, A. (2017). Study on the growth and spatial distribution of the root system of different european pear cultivars on quince rootstock combinations. Revista Brasileira de Fruticultura, 40(2): 7 p. https://doi.org/10.1590/0100-29452018108.

Malamy, J. E. (2005). Intrinsic and environmental response pathways that regulate root system architecture. Plant, cell \& environment, 28(1): 67-77. https://doi.org/10.1111/j.1365-3040.2005.01306.x.

Milandinovic, V. (2016). Tree protection policy and specifications for construction near trees. Toronto, Canadá. 18 p. En: https://www.toronto.ca/data/parks/pdf/trees/tree-protection-specs.pdf.

Ministerio de Agricultura. (1995). Decreto 13. Declara Monumento Natural las Especies Forestales Queule, Pitao, Belloto del Sur, Belloto del Norte y Ruil. Santiago. 2 p. 
Ministerio Secretaría General de la Presidencia, (2008). Aprueba y oficializa nómina para el segundo proceso de clasificación de especies según su estado de conservación. $\mathrm{N}^{\circ} 50$. Diario Oficial de la República de Chile $\mathrm{N}^{\circ}$ 39.100. Chile.

Moore, W. (2003). El modelo de las zonas del árbol. Arbres et Sciences, 8(2): 37p.

Morales, N., Becerra, P., Arellano, E. \& Gilabert, H. (2015). Effect of large and small herbivores on seed and seedling survival of Beilschmiedia miersii in central Chile. Bosque, 36(1): 127-132. http://dx.doi.org/10.4067/S071792002015000100014.

Moreno, M., Pineda, J., Colinas, M. T. \& Sahagún, J. (2020). El oxígeno en la zona radical y su efecto en las plantas. Revista Mexicana de Ciencias Agrícolas, 11(4): 931-943.

Mulia, R., y Dupraz, C. (2006). Unusual fine root distributions of two deciduous tree species in southern France: what consequences for modelling of tree root dynamics?. Plant and Soil, 281(1): 71-85. https://doi.org/10.1007/s11104-005-3770-6.

Novoa, P. (2004). Determinación del grado de amenaza del belloto del norte (Beilschmiedia miersii Kosterm, Lauraceae), mediante el uso de la metodología UICN 2001. Versión 3.1. Chloris Chilensis Año 7 № 2. URL: http://www.chlorischile.cl. Basado en el Documento Técnico $N^{0} 387$ de la Oficina de Estudios y Planificación, CONAF - V Región. En: http://www.chlorischile.cl/bellotonovoa/bellotopaper.htm.

Olmo, M., Lopez-Iglesias, B. \& Villar, R. (2014). Drought changes the structure and elemental composition of very fine roots in seedlings of ten woody tree species. Implications for a drier climate. Plant and Soil, 384(1): 113-129. https://doi.org/10.1007/s11104-014-2178-6.

Ovalle, J., Arellano, A. \& Rossana G. (2015). Trade-Offs between Drought Survival and Rooting Strategy of Two South American Mediterranean Tree Species: Implications for Dryland Forests Restoration. Forests, 6(10): 37333747. https://doi.org/10.3390/f6103733.

Padilla, F.M. \& Pugnaire, F.I. (2007). Rooting depth and soil moisture control Mediterranean woody seedling survival during drought. Functional Ecology, 21(3): 489-495. https://doi.org/10.1111/j.1365-2435.2007.01267.x.

Pallant, E., Holmgren, R.A., Schuler, G.E., McCracken, K.L. \& Drbal, B. (1993). Using a fine-root extraction device to quantify small-diameter corn roots $(\leq 0.025 \mathrm{~mm})$ in field soils. Plant and Soil, $153 . \quad \mathrm{Pp}$ : $273 \quad-$ 279. https://doi.org/10.1007/BF00013000.

Perry, T. (1989). Tree Roots: Facts and Fallacies. Arnoldia, 49(4): 3-29.

Pickles, B. \& Simard, S. (2017). Chapter 18 - Mycorrhizal networks and forest resilience to drought. En: Collins, N; Gehring, C y Jansa, J. (Eds). Mycorrhizal mediation of soil: Fertility, Structure, and Carbon Storage. Elsevier. Pp: 319-339. https://doi.org/10.1016/B978-0-12-804312-7.00018-8.

Pierret, A., Moran, C.J. \& Doussan, C. (2005). Conventional detection methodology is limiting our ability to understand the roles and functions of fine roots. New Phytologist, 166. Pp: 967-980. https://doi.org/10.1111/j.14698137.2005.01389.x.

Pierret, A., Maeght, J., Clément, C., Montoroi, J., Hartmann, C., \& Gonkhamdee, S. (2016). Understanding deep roots and their functions in ecosystems: an advocacy for more unconventional research. Annals of botany, 118(4): 621-635. https://doi.org/10.1093/aob/mcw130.

Poole, D.K. \& Miller, P.C. (1978). Water-related characteristics of some evergreen sclerophyll shrubs in central Chile. Oecologia Plantarum, 13. Pp: 289-299.

Rizzardi, S. \& Calvo, G. (2019). Sistema radicular de los árboles en ámbitos urbanos. Terra Mundus, 5(1): 1-23.

Rodríguez, R., Mattei, O. \& Quezada, M. (1983). Flora arbórea de Chile. Editorial de la Universidad de Concepción. Concepción, Chile. Pp: 83-85.

Rundel, P.W., Sharifi, M.R., Vu, M.K., Montenegro, G., \& Mooney, H. (2019). A Seasonal patterns of growth phenology and nutrient dynamics in four matorral shrubs in Central Chile. Gayana Botánica, 76(2): 208-219. http://dx.doi.org/10.4067/S0717-66432019000200208.

Sanesi, G., Lafortezza, R., Colangelo, G., Marziliano, P. \& Davies, C. (2013). Root system investigation in sclerophyllous vegetation: an overview. Italian Journal of agronomy, 8(2): $121-126$. https://doi.org/10.4081/ija.2013.e17.

Serra, M. T., Gajardo, R. \& Cabello, Á. (1986). Beilschmiedia miersii. Programa de protección y recuperación de la flora nativa de chile. Ficha técnica de especies amenazadas. Corporación Nacional Forestal (CONAF). 23 p. 
Schenk, H.J. \& Jackson, R.B. (2002). The global biogeography of roots. Ecol. Monogr., 72(3): $311-328$. https://doi.org/10.1890/0012-9615(2002)072[0311:TGBOR]2.0.CO;2.

Simard, S. (2012). Mycorrhizal networks and seedling establishment in Douglas-fir forests (Chapter 4). En: Southworth, Darlene (Ed.). Biocomplexity of Plant-Fungal Interactions, First Edition. London: John Wiley \& Sons, Inc. Pp: 85-107. https://doi.org/10.1002/9781118314364.ch4.

Simard, S., Asay, A.K., Beiler, K.J., Bingham, M.A., Deslippe, J.R., He, X., Philip, L.J., Song, Y. \& Teste, F.P. (2015). Resource transfer between plants through ectomycorrhizal networks. En: Horton, TR (Ed.). Mycorrhizal networks, Ecological studies (Analysis and Synthesis), vol. 224. Springer, Dordrecht. Pp: $133-176$. https://doi.org/10.1007/978-94-017-7395-9.

Sponchiado, B.N., White, J.W., Castillo, J.A, \& Jones, P.G. (1989). Root growth of four common bean cultivars in relation to drought tolerance in environments with contrasting soil types. Experimental Agriculture, 25(2): $249-257$. https://doi.org/10.1017/S0014479700016756.

Thomas, P. (2014). Trees: their natural history Trees Their Natural History. Chapter 4: Roots: the hidden tree. (How far do roots spread?). Second edition. Keele University, UK. Cambridge University Press. United Kingdom. 110 p. https://doi.org/10.1017/CBO9781139026567.

Verdaguer, D. \& Molinas, M. (1992). Anatomía y caracterización del sistema radicular del alcornoque. Scientia gerundensis, 18. Pp: 39-51.

Watson, A. \& O'Loughlin, C. (1990). Structural root morphology and Biomass of three age-classes of Pinus radiata. New Zealand Journal of Forestry Science, 20(1): 97-110. ISSN: 0048-0134 
Ciencia \& Investigación Forestal Vol. 27 № 2. Agosto 2021 